\title{
An Unusual Case of Pulmonary Alveolar Microlithiasis Masquerading as Pulmonary Tuberculosis
}

\author{
Nadia llyas ${ }^{1}$, Sandhya Poflee ${ }^{2}$, Vedita Bobde ${ }^{3}$, Satish Helwatkar ${ }^{3}$ \\ ${ }^{1} J u n i o r$ Resident (Pathology), Department of Pathology, Government Medical College and Hospital, Nagpur, ${ }^{2}$ Associate Professor, Department of Pathology, \\ Government Medical College and Hospital, Nagpur, ${ }^{3}$ Assistant Professor, Department of Pathology, Government Medical College and Hospital, Nagpur.
}

\section{Abstract}

Pulmonary Alveolar Microlithiasis (PAM) is a rare chronic lung disease characterized by bilateral intra-alveolar calcium and phosphate deposition throughout the lung parenchyma. PAM is known to occur over wide age range. Exact etiopathogenesis of PAM is not known and long term survival is uncertain. Patient can be asymptomatic or present with progressive pulmonary symptoms. Radiological features of PAM are pathognomic for radiologist who is aware of this rare pulmonary disease. However the changes of PAM on imaging can be misdiagnosed as those due to pulmonary tuberculosis which is a relatively common diagnosis in the Indian setting. Definitive diagnosis is based on typical microscopic features seen on transbronchial or open lung biopsy. We report a case of 41 years old female who was treated as a case of pulmonary tuberculosis for two years without relief of symptoms at a peripheral medical centre. Final diagnosis of PAM was made on the basis of characteristic radiological findings and microscopic features at our Institute.

Keywords: Pulmonary Alveolar Microlithiasis, Imaging findings in PAM, Histopathological features of PAM.

Corresponding Author: Dr. Sandhya V Poflee, 203, Imperial Regal, Plot no. 545, Ramdaspeth, Nagpur-440010, Maharashtra, India.

Received: November 2019

Accepted: November 2019

\section{Introduction}

Pulmonary alveolar microlithiasis is an uncommon disease that produces characteristic $\mathrm{x}$-ray chest findings due to intra-alveolar accumulation of minute calcium calculi in both lungs. ${ }^{[1]}$ Till now about 1042 cases have been reported worldwide. First case from India was reported by Vishwanathan in 1962 and 86 cases have been reported since then. ${ }^{[2]}$ PAM presents with clinical symptoms of varied severity that at times mimic those of more common disease like tuberculosis. ${ }^{[3]}$ The case reported here was initially referred to Chest Medicine Department of Tertiary Care Hospital as that of Multi Drug Resistant Tuberculosis (MDR-TB) with superadded acute lung infection. As the treatment varies for PAM and its close differential diagnoses, it is essential to identify this rare condition.

\section{Case Report}

41 year old female presented to Chest Medicine Department of Tertiary Care Hospital from central India with complaints of shortness of breath since 2 months. Her other complaints were productive cough and low grade fever of 1 month. She had generalized weakness for the same duration.

Two years back she was empirically given Anti- Koch's Treatment for nine months from a rural medical centre on the basis of similar complaints. Even on completion of her
MDR-TB regimen there was no significant relief from her symptoms. She is a farmer by occupation for last 20 years. She did not give history of any addictions but gave history of household fuel smoke exposure for the last 25 years. Her family history was not significant.

On general examination patient was moderately built adult female with stable vitals and systemic examination was mostly within normal limits. Respiratory examination revealed bilateral crepts with $\mathrm{SpO} 294 \%$ on room air.

Complete blood count showed moderate anemia with hemoglobin $8.9 \mathrm{~g} / \mathrm{dl}$ and raised total leucocyte count 20,000 cells/mm3 with leukocytosis and mild shift to left. Sputum cytology was negative for abnormal cells, sputum CBNAAT (Cartridge Based Nucleic Acid Amplifiation Test) was negative and Acid fast Tubercular bacilli were not detected. Smears prepared from bronchoalveolar lavage showed abundant macrophages with the background showing densely basophilic granular material. It was negative for fungal elements and no organisms were detected on Gram stain. Patient was tested for HIV by ELISA technique and found to be negative for it.

On imaging, postero-anterior view of chest X-ray revealed characteristic sand storm appearance with multiple sand like calcifications present throughout the lung parenchyma involving bilaterally. [Figure 1a] HRCT thorax revealed extensive subpleural, parenchymal and peribronchial calcifications in bilateral lung fields. Interlobular septal thickening with characteristic crazy paving appearance was observed. [Figure 1b] 


\section{Ilyas et al; An Unusual Case of Pulmanary Alvealar Micralithiasis Masquerading as Pulmanary}

Tuberculasis

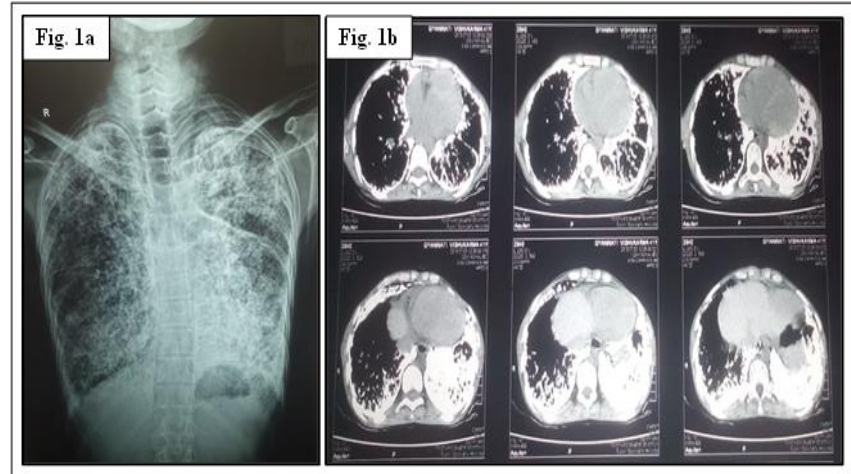

Figure 1: Radiological images showing (a) Characteristic "sand storm" appearance on chest radiograph and (b) "Crazy paving" appearance on HRCT.

Oesophago-gastric-duodenoscopy study was done and was normal. No calcification was detected on CT scan of abdomen. Based on radiological findings, differential diagnosis of Pneumoconiosis and Pulmonary Alveolar Microlithiasis were considered and transbronchial lung biopsy was performed. Single tiny grey white tissue of size $0.3 \times 0.2 \times 0.1 \mathrm{~cm}$ was sent to histopathology section of Pathology department of the Institute. The complete tissue was paraffin embedded and subjected to routine processing and H\&E stained. Microscopic examination of the biopsy revealed bronchial cartilage and multiple alveolar spaces filled with calcific material with peripheral concretions. Adjacent fibrocollagenous tissue showed infiltration by acute and chronic inflammatory cell infiltrate. [Figure $2 \mathrm{a} \&$ 2b]. No evidence of tubercular granuloma was seen. Histopathological findings confirmed the radiological possibility of Pulmonary Alveolar Microlithiasis.
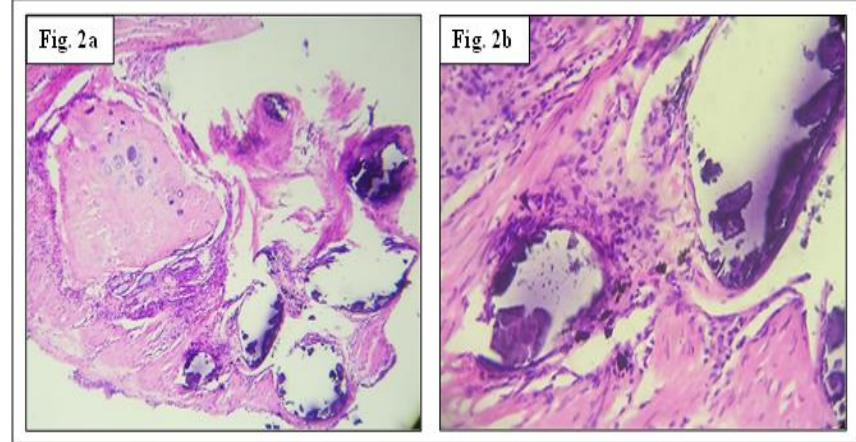

Figure 2: Transbronchial biopsy showing (a) Bronchial cartilage and multiple alveolar spaces filled with calcific material (H\&E 10X) and (b) Infiltration of adjacent fibrocollagenous tissue by inflammatory cells (H\&E 40X).

Chest X-ray of her sister and two sons were within normal limits. So a diagnosis of sporadic Pulmonary Alveolar Microlithiasis was considered in this case. Patient was given a course of antibiotics for the superadded infection and nebulized with bronchodilators. On resolution of acute symptoms the patient was discharged and advised long-term follow-up.

\section{Discussion}

Pulmonary alveolar microlithiasis (PAM) is an autosomal recessive metabolic interstitial lung disease. It presents worldwide in sporadic as well as familial occurrence with a slightly higher incidence in Asian countries. Till June 2018 $\neg, 86$ cases were reported from India and 1042 cases reported worldwide. ${ }^{[2]}$ It is characterized by deposition of intra-alveolar calcium and phosphate throughout the lung parenchyma. The etiopathogenesis of PAM is not very well understood however the SLC34A2 gene coding for the sodium-phosphate co-transporter in type II pneumocytes is deemed responsible for the disease. Mutation in the gene leads to decreased clearance of calcium and phosphate from alveoli resulting in formation of laminated microliths or calcispherites. ${ }^{[4]}$ Microliths range from 50 to $1000 \mathrm{~mm}$ and are composed predominantly of calcium and phosphate with a small component of iron, magnesium, potassium and copper. Extrapulmonary calcifications have also been reported in other organs such as kidney, prostate, pancreas, ovary, uterus, testis, thyroid gland, salivary gland, trachea and placenta. ${ }^{[3]}$

PAM is a progressive disease showing asynchrony between the severity of clinical presentation and radiological findings. ${ }^{[5]}$ No significant sex predilection has been noted among familial cases but sporadic cases show slight male predominance. Diagnosis of PAM most commonly occurs in third or fourth decade, however, the disease may manifest at any age. ${ }^{[6]}$

Initially the disease is asymptomatic and often the diagnosis of PAM is made incidentally on chest radiography done for other reasons. ${ }^{[3]}$ Serum calcium and phosphate concentrations and hepatic, renal, and parathyroid values, are normal. As the disease progresses manifestation of respiratory symptoms such as dry cough, dyspnoea, clubbing, cyanosis, chest pain and weakness are evident. In later stages there may be pulmonary hypertension, respiratory and cardiac arrest and ultimately death. Due to the chronicity of the disease, long term follow up of patients is rarely obtained.

Thorough clinical history, physical examination and characteristic radiological findings most often clinch the diagnosis of PAM. Histopathological examination of lung biopsy and bronchoscopic BAL are required for confirmation. ${ }^{[3]}$ Diagnostic sensitivity of genetic testing for mutations in the SLC34A2 gene is not yet established. Presence of this genetic mutation in patient I considered contributory for the diagnosis of PAM. ${ }^{[7]}$ Calcific sand like micro nodules involving the middle and lower zones of both lungs predominantly give a typical 'sand storm' appearance on chest X-ray. ${ }^{[5]}$ HRCT scan shows diffuse micronodules with slight perilobular predominance. Calcification occurring at the periphery of secondary pulmonary lobules results in interstitial septal thickening thus giving the characteristic 'crazy paving' pattern. ${ }^{[8]}$ 'Black pleura' sign is also seen which represents subpleural sparing of pulmonary calcification. ${ }^{[9]}$

Differential diagnoses of PAM include miliary tuberculosis, 
pulmonary hemosiderosis, sarcoidosis, pneumoconiosis, amyloidosis, and metastatic pulmonary calcifications associated with chronic renal failure and hemodialysis. ${ }^{[8]}$ In our case the differential diagnoses of pneumoconiosis and PAM were given on radiology. In contrast to PAM, HRCT in simple coal workers pneumoconiosis typically reveals small $2-5 \mathrm{~mm}$ centrilobular and subpleural circumscribed nodules predominantly in upper lobes but can involve the entire lung parenchyma diffusely. ${ }^{[10]}$ On microscopic examination, coal macules around respiratory bronchioles are the characteristic finding of pneumoconiosis comprising of carbon-containing macrophages surrounded by a network of collagen fibers and fibroblasts. Calcifications can form in $10 \%-20 \%$ of patients. ${ }^{[10]}$ Histopathological examination of the transbronchial biopsy obtained in our case did not show any of the mentioned features.

Histopathological examination in a case of PAM is characterized by presence of microliths or calcospherites within the alveoli. ${ }^{[1]}$ Transbronchial biopsy is necessary to rule out such close differential diagnoses. As in our case Pneumoconiosis and Tuberculosis were ruled out on histopathological examination.

No definite treatment has yet been established for PAM although various trials of disodium etidronate, corticosteroids, therapeutic bronchoalveolar lavage and gene therapy are ongoing ${ }^{[5]}$ Lung transplantation is the only definite treatment to be considered in PAM ${ }^{[6]}$ Supplemental oxygen therapy and pneumococcal vaccinations are also recommended for these patients. ${ }^{[5]}$

\section{Conclusion}

We conclude that Pulmonary Alveolar Microlithiasis, although a rare disease, should be considered as a differential diagnosis in cases of non-responding pulmonary infections especially Tuberculosis. Early diagnosis of this rare entity lies primarily with the radiologist and confirmation by histopathology is often essential.

\section{Acknowledgement}

We acknowledge the Departments of Chest Medicine and Radiodiagnostics and Professor and Head, Department of Pathology, Government Medical College and Hospital, Nagpur for their contribution to the case.

\section{References}

1. Myers JL. Lung. In: Goldblum JR, Lamps LW, McKenney JK, Myers JL. Rosai and Ackerman's Surgical Pathology. 11th ed. Elsevier 2018. p.398-99.

2. Chopra M, Tendolkar MS, Vardhan V. BMJ Case Rep 2019;12:e227406. DOI:10.1136/bcr-2018227406.

3. Lina M, Jonsson A, Simonsen U, Hilberg O, Bendstrup E. Pulmonary alveolar microlithiasis: two case reports and review of the literature. European Respiratory Review. 2012;21(125):249-256.

4. Narahari NK, Vuhini SK. Pulmonary Alveolar Microlithiasis: A Retrospective study. Sch J App Med Sci 2016; 4(6D):2102-07.

5. Stamatopoulos A, Patrini D, Mitsos S, Khiroya R, Borg E, Hayward M et al. An unusual late onset of pulmonary alveolar microlithiasis: A case report and literature review. Respiratory Medicine Case Reports. 2017;22:24-27.

6. Arpağ H, Sayan M, Atilla N, Bozkus F, Bahar AY, Kahraman H, et al. A Case of Pulmonary Alveolar Microlithiasis Diagnosed by Transbronchial Biopsy. Turk Thorac J 2017;18:134-6.

7. Mehta K, Dell S, Birken C, Al-Saleh S. Case report: Pulmonary Alveolar Microlithiasis. Can Resp J [Internet]. 2016;Article ID 4938632, 4 pages, 2016. Available from: https://doi.org/10.1155/2016/4938632.

8. Delik JA, Fuhrman CR, Bittar HET. Pulmonary Alveolar Microlithiasis. RadioGraphics 2016; 36:1334-38.

9. Kashyap S, Mohapatra PR. Pulmonary alveolar microlithiasis. Lung India 2013;30:143-7.

10. Shah SH, Nagymanyoki Z, Ramaiya NH, Howard S. AIRP Best Cases in RadiologicPathologic Correlation Coal Workers' Pneumoconiosis. RadioGraphics 2012; 32:2047-52.

Copyright: () the author(s), 2019. It is an open-access article distributed under the terms of the Creative Commons Attribution License (CC BY 4.0), which permits authors to retain ownership of the copyright for their content, and allow anyone to download, reuse, reprint, modify, distribute and/or copy the content as long as the original authors and source are cited.

How to cite this article: Ilyas N, Poflee S, Bobde V, Helwatkar S. An Unusual Case of Pulmonary Alveolar Microlithiasis Masquerading as Pulmonary Tuberculosis. Asian J. Med. Res. 2019;8(4):PT01-PT03.

DOI: dx.doi.org/10.21276/ajmr.2019.8.4.PT1

Source of Support: Nil, Conflict of Interest: None declared. 